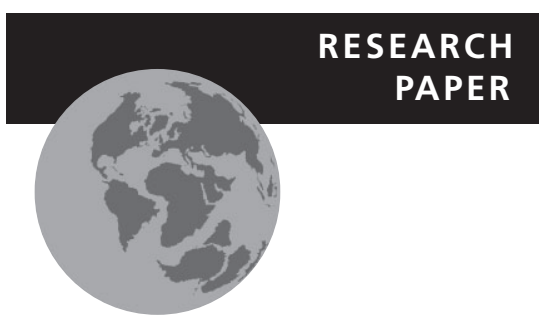

\section{Late bloomers and baby boomers: ecological drivers of longevity in squamates and the tuatara}

\author{
Inon Scharf $^{1 \star}$, Anat Feldman ${ }^{1}$, Maria Novosolov $^{1}$, Daniel Pincheira-Donoso ${ }^{2}$, \\ Indraneil Das ${ }^{3}$, Monika Böhm ${ }^{4}$, Peter Uetz ${ }^{5}$, Omar Torres-Carvajal ${ }^{6}$, \\ Aaron Bauer ${ }^{7}$, Uri Roll ${ }^{8}$ and Shai Meiri ${ }^{1 \star}$
}

${ }^{1}$ Department of Zoology, Faculty of Life Sciences, Tel Aviv University, Tel Aviv, Israel, ${ }^{2}$ Laboratory of Evolutionary Ecology of Adaptations, School of Life Sciences, University of Lincoln, Lincolnshire, UK, ${ }^{3}$ Institute of Biodiversity and Environmental Conservation, Universiti Malaysia Sarawak, Kota Samarahan, Malaysia, ${ }^{4}$ Institute of Zoology, Zoological Society of London, London, UK, ${ }^{5}$ Center for the Study of Biological Complexity, Virginia Commonwealth University, Richmond, VA, USA, ${ }^{6}$ Escuela de Ciencias Biológicas, Pontificia Universidad Católica del Ecuador, Apartado 17-01-2184, Quito, Ecuador, ${ }^{7}$ Department of Biology, Villanova University, Villanova, PA, USA, ${ }^{8}$ School of Geography and of the Environment, University of Oxford, UK

\footnotetext{
${ }^{*}$ Correspondence: Inon Scharf and Shai Meiri, Department of Zoology, Faculty of Life Sciences, Tel Aviv University, 69978 Tel Aviv, Israel.

E-mails: scharfi@post.tau.ac.il (IS); uncshai@post.tau.ac.il (SM)

The first and second authors contributed equally to this work.
}

\begin{abstract}
Aim Longevity is an important life-history trait, directly linked to the core attributes of fitness (reproduction and survival), yet large-scale comparative studies quantifying its implications for the ecology and life history of ectotherms are scarce. We tested the allometry of longevity in squamates and the tuatara, and determined how longevity is related to key environmental characteristics and lifehistory traits. Predictions based on life-history theory are expected to hold true for ectotherms, similarly to mammals and birds.
\end{abstract}

Location World-wide.

Methods We assembled from the literature a dataset of the maximum longevities of more than a thousand squamate species, representing c. 10\% of their known species diversity, their phylogenetic relationships and multiple life-history and ecological variables. Correcting for phylogeny, we modelled the link between squamate longevity and both key life-history traits, such as body mass and age at first reproduction, and important environmental factors, such as latitude and primary productivity within species distributional ranges.

Results Large-bodied species live for longer than small ones, but body size explains far less of the variance in longevity than it does in mammals and birds. Accounting for body size, squamate brood frequency is negatively correlated with longevity, while age at first reproduction is positively correlated with longevity. This points to a continuum of slow-to-fast life-history strategies. Squamates in high latitudes and cold regions live for longer, probably because a shorter season of activity translates to slower development, older age at first reproduction and hence to increased longevity. Individuals live longer in captivity than in the wild. Herbivorous and omnivorous squamates live for longer than carnivorous ones. We postulate that low-quality nutrition reduces growth rates, promotes a relative decline in reproductive rates and thus prolongs life.

Main conclusions Our results support key predictions from life-history theory and suggest that reproducing more slowly and at older ages, being herbivorous and, plausibly, lowering metabolism, result in increased longevity.

\section{Keywords}

Body size, fast-slow continuum, lifespan, NPP, phylogenetic comparisons, reproduction, reptiles, temperature, trade-off. 